\title{
Influencia del Apoyo Educativo en las Capacidades de Autocuidado del Adulto Mayor del Centro Gerontológico Integral de Tlahuelilpan Hidalgo
}

\author{
Influence of educational support on the self-care abilities of the elderly in the Integral \\ Gerontological Center of Tlahuelilpan Hgo. \\ Claudia A. Tejo García ${ }^{a}$, Gabriela Maldonado Muñiz ${ }^{b}$
}

\begin{abstract}
:
Today, life expectancy has increased to the age of 60 and over. During aging, self -care abilities are diminished. However, through educational strategies they can be preserved and maintained, allowing them to have a full and functional life. Objective: To evaluate the influence of educational support on the self-care capabilities of the elderly within the Integral Gerontological Center of Tlahuelilpan Hgo. Material and Methods: Quantitative research, quasi-experimental design with pre- test and post - test. Sample made up of 27 older adults from the Integral Gerontological Center of Tlahuelilpan Hgo. The Self- Care Agency (ASA) Appreciation Scale consisting of 24 items was used, with a response format of four Likert alternatives, where the score ranges from 24 to 96 points, reliability by Alpha de Crombach of 0.70 . Results: Of the study population, $74 \%$ belong the female gender, $67 \%$ have primary education, $63 \%$ are married and $74 \%$ are dedicated to the home.
\end{abstract}

Regarding self-care abilities, it was found that $93 \%$ of older adults have good self - care abilities; afterthe intervention, $89 \%$ increased to very good capacities, and $11 \%$ to good capacities.

Keywords:

Abilities, Self-care, Elderly, Educational Support

\section{Resumen:}

En la actualidad la esperanza de vida se ha incrementado hasta entrados los 60 años y más. Durante el envejecimiento las capacidades de autocuidado se ven disminuidas, Sin embargo, a través de estrategias educativas se pueden preservar y mantener, permitiendo tener una vida plena y funcional. Objetivo: Evaluar la influencia del apoyo educativo en las capacidades de autocuidado del adulto mayor dentro del Centro Gerontológico Integral de Tlahuelilpan Hgo. Material y Métodos: Investigación de tipo cuantitativa, diseño cuasi experimental con preprueba y posprueba. Muestra conformada por 27 adultos mayores del Centro Gerontológico Integral de Tlahuelilpan Hgo. Se utilizó la escala de Apreciación de la Agencia de Autocuidado (ASA) constituida por 24 items, con un formato de respuestas de cuatro alternativas Likert, en donde la puntuación va de 24 a 96 puntos, confiabilidad por Alpha de Crombach de 0.70. Resultados: De la población en estudio $74 \%$ pertenecen al género femenino, $67 \%$ tiene estudios de primaria, $63 \%$ son casados y $74 \%$ se dedica al hogar.

En cuanto a las capacidades de autocuidado se encontró que el 93\% de los adultos mayores tienen buenas capacidades de autocuidado, posterior a la intervención $89 \%$ aumentaron a muy buenas capacidades, y el $11 \%$ a buenas capacidades.

Palabras Clave:

Capacidades, Autocuidado, Adulto Mayor, Apoyo Educativo

autor de Correspondencia, Profesor Investigador de Universidad Autónoma del Estado de Hidalgo, Escuela Superior de Tlahuelilpan, ORCID: https://orcid.org/0000-0003-2205-7879,Email: ctrejo@uaeh.edu.mx

b Universidad Autónoma del Estado de Hidalgo, Escuela Superior de Tlahuelilpan, ORCID: https://orcid.org/0000-0002-4967-1812, Email: gmaldonado@uaeh.edu.mx 


\section{Introducción}

El envejecimiento de la población mundial es un fenómeno al cual muchos países están tratando de adaptarse, lo que en el pasado era una característica de algunas naciones, paso a ser una experiencia creciente en todo el mundo. Las causas para la ocurrencia de este fenómeno se relacionan con varios factores, entre los cuales cabe mencionar: loa avances médicos, aumento de la expectativa de vida de la población y la calidad de vida. (Garcilazo Salazar, 2019)

El incremento de la esperanza de vida en México representa uno de los mayores retos a los que actualmente se enfrenta la sociedad. México se encuentra en un proceso de envejecimiento natural de la población, los mayores de 65 años llegaron a 8.5 millones de habitantes, monto que reportó un crecimiento de $23.2 \%$ en comparación con los datos del Censo Poblacional. (Organización Mundial de la Salud, 2015)

La importancia de conocer los cambios que se generan en el adulto mayor es la de identificar aquellos que pueden comprometer la capacidad de autocuidado del adulto mayor. La limitación para desarrollar los roles y actividades en un ambiente definido es una de las principales consecuencias, pudiendo afectar el ámbito personal, laboral y social dificulta la forma en la que el adulto mayor pueda afrontar todos los cambios propios de la edad que pasa su organismo, aunado a los que generan las enfermedades crónico degenerativas.

Orem menciona que el profesional de enfermería debe ser capaz de identificar las necesidades de las personas que atiende, elegir el método de ayuda a implementar con los el individuo para que sus requisitos de autocuidado puedan ser atendidos (Raile , 2018)

La teoría de Orem es una teoría general integrada portres subteorías relacionadas:

- Teoría del déficit de autocuidado

- Teoría del autocuidado

- Teoría de sistemas

Para esta investigación se retomó la teoría de sistemas la cual explica los modos en que las enfermeras/os pueden atender a los individuos, específicamente es sistema de apoyo educativo, en donde enfermería actúa ayudando a los individuos para que estos sean capaces de realizar las actividades de autocuidado.

Por lo que el objetivo de esta investigación es evaluar la influencia del apoyo educativo en las capacidades de autocuidado del adulto mayor del Centro Gerontológico Integral de Tlahuelilpan Hgo.,

\section{Planteamiento del problema o exposición del caso}

El envejecimiento es un proceso natural e irreversible, que comprende cambios biopsicosociales en el individuo. Estos cambios conducen al deterioro funcional y pérdida se la iniciativa, lo que genera vulnerabilidad y por ende disminución del bienestar. Es necesario que el adulto mayor logre una longevidad satisfactoria evidenciada en su calidad de vida, por tanto, conseguir una mejor calidad de vida del adulto mayor implica asumir voluntariamente el cuidado de su propia salud, es decir practicar el auto cuidado, lo que ayudará a mantener un envejecimiento seguro y saludable. (Garcilazo Salazar, 2019)

El proceso de envejecimiento repercute en varios factores del desarrollo y funcionamiento de la sociedad; el envejecimiento saludable debe abordarse no sólo desde la perspectiva médica sino con una óptica integral, es necesario atender los aspectos clínicos, sociales, económicos y culturales

El estado de salud del adulto mayor suele ser el reflejo o resultado de hábitos saludables que se han tomado durante el transcurso de la vida. En esta etapa del ciclo vital, el proceso de envejecimiento es clave como proceso natural que genera una serie de cambios estructurales y funcionales, los cuales influyen el estado físico, psicológico, social y a su vez incide en la capacidad de autocuidado.

Para la enfermería, el eje fundamental y objeto de conocimiento, del saber y del quehacer es el cuidado de la salud, el cual es un proceso cuyo fin es brindar cuidado integral de salud a la persona, familia y comunidad, ayudando a desarrollar al máximo los potenciales individuales y colectivos, para mantener prácticas salu dables que permitan salvaguardar el estado óptimo de salud en todas las etapas de la vida. (Castiblanco Amaya \& Fajardo, 2017)

La Teoría de Orem se fundamenta en premisas en donde los individuos tienen la potencialidad para desarrollar sus habilidades y prácticas y la motivación esencial para el autocuidado, partien do de ellas enfermería puede apoyar a los adultos mayores para que satisfagan sus necesidades de autocuidado, ayu dándoles a desarrollar y ejercer sus capacidades.

Por lo que surge la siguiente interrogante:

¿Cuál es la influencia del apoyo educativo en las capacidades de autocuidado del adulto mayor del Centro Gerontológico Integral de Tlahuelilpan Hidalgo? 


\section{Conocimiento previo}

Actualmente, la mayor parte de la población tiene una esperanza de vida igual o superior a los 60 años. Para 2050, se espera que la población mundial en esa franja de edad llegue a los 2000 millones, un aumento de 900 millones con respecto a 2015. (Organización Mundial de la Salud, 2018)

En búsqueda bibliográfica se encontraron artículos de interés que aportan a la investigación, entre los cuales se encuentran el de Castiblanco Amaya y Fajardo en sus estudios capacidad y percepción de autocuidado del adulto mayor del programa Centro día/vida, Ibagué, Colombia, en donde el análisis general demostró que el adulto mayor presenta una adecuada capacidad y percepción de autocuidado.

\section{Metodología de diseño o de estudio}

Investigación de tipo aplicada, con enfoque cuantitativo, longitudinal, de diseño pre - experimental preprueba/post prueba, con un solo grupo. El universo estuvo constituido por 27 adultos mayores del Centro Gerontológico Integral de Tlahuelilpan Hgo.

Previa autorización de la unidad y del consentimiento otorgado por los participantes del estudio, se aplicó la escala de apreciación de la capacidad de la agencia de autocuidado (ASA), integrada por 24 ítems, con un formato de respuesta de 4 alternativas tipo Likert, donde $1=$ nuca (valor más bajo de la capacidad de autocuidado) y 4 = siempre (valor más alto), cada individuo puede obtener puntajes de van desde 24 a 96 puntos, teniendo como capacidades de autocuidado: muy bajas (1-24 pts.) bajas ( 25 - 48 pts.), buenas (49 -72 pts.) y muy buenas (73 a 96 pts.), escala con una confiabilidad por Alfa de Cronbach de 0.70

Se implemento el apoyo educativo mediante el programa: "Cuidándome para estar mejor", desarrollado en 6 sesiones educativas, con énfasis en autocuidado de acuerdo a los déficits identificados en la primera medición, cada sesión tuvo una duración de 90 minutos.

Se utilizó estadística descriptiva e inferencial, prueba de rangos de Wilcoxon con el programa de Excel de Microsoft y el paquete estadístico SPSS.

\section{Aplicación}

Para esta investigación se retomó la teoría de sistemas la cual explica los modos en que las enfermeras/os pueden atender a los individuos, específicamente es sistema de apoyo educativo, en donde enfermería actúa ayudando a los individuos para que estos sean capaces de realizar las actividades de autocuidado.

\section{Resultados}

En relación a los factores condicionantes básicos de la población en estudio se encontró que:

El promedio de edad de los adultos mayores fue de 70.48 \pm 6.00 , siendo el rango de edad más frecuente el de 73 a 78 años.

El $74 \%(n=20)$ del universo de estudio son del género femenino y $26 \%$ ( $n=7)$ masculino. Respecto al nivel de escolaridad el $67 \%(n=18)$ tienen educación primaria, $22 \%$ $(n=6)$ son analfabetas y solamente el $11 \%(n=3)$ tienen estudios de secundaria.

El estado civil predominante en los adultos mayores es el de casado con $63 \%(n=17), 22 \%(n=6)$ son viudos y el $15 \%(n=4)$ son solteros.

El 74\% ( $n=24)$ se dedica al hogar, 19\% $(n=5)$ se dedica a la agricultura y el $7 \%(n=2)$ no tiene ocupación.

En la pre prueba de las capacidades de autocuidado se encontró que el $93 \%(n=25)$ de los adultos mayores del Centro Gerontológico Integral de Tlahuelilpan Hgo. tienen buenas capacidades de autocuidado, solamente el $7 \%$ $(n=2)$ evidencia bajas capacidades

Una vez implementado la obtención de resultados pos interven ción se obtuvo un considerable cambio, donde se encontró que el $89 \%(n=24)$ donde la población posee muy buenas capacidades de autocuidado, el $11 \%(n=3)$ de ella se ubica en buenas capacidades descartando así las bajas y muy bajas capacidades.

La prueba de rangos de Wilcoxon para una muestra refiere que de acuerdo a la comparación de dos resultados (pre y post) debe obtener un nivel de significancia de $p \leq 0.05$ para aceptar la hipótesis de trabajo, en este caso se obtuvo $z=-4.9 p=0.000$, por lo que se aprueba la hipótesis de trabajo: El apoyo educativo aumenta la capacidad de autocuidado del adulto mayor del Centro Gerontológico de Tlahuelilpan Hgo.

\section{Discusión}

Se observó que la edad promedio de la población estudiada radica entre $70.48 \pm 6.00$ años siendo el rango de edad más frecuente entre 73 a 78 años, lo que es un factor para que el adulto mayor tenga menor capacidad de autocuidado Concordando con la investigación realizada por Puello Cecilia Elsyen Obtuvo como resultados que el $48 \%$ de la población estaba en el rango etario entre 67 y 72 años, por lo que existe una amplia comparación afirmativa en 
cuanto a este estudio al notar en nuestros resultados que entre mayor edad mayor déficit de autocuidado.

Respecto a la capacidad de autocuidado del adulto mayor se encontró que el $89 \%(n=24)$ tienen muy buenas capacidades de autocuidado posteriores a una intervención educativa estos resultados; situación también observada por Castiblanco Amaya en el cual determinó que la capacidad y percepción de autocuidado de los adultos mayores es parcialmente adecuada en donde se evidencio déficit de autocuidado inadecuado para ninguna categoría, debido a la interdisciplinariedad del programa.

El apoyo educativo es una herramienta fundamental para lograr en los adultos mayores mejores capacidades de autocuidado y así encaminarlos a un envejecimiento saludable.

\section{Conclusiones}

Orem establece que el autocuidado es una conducta aprendida que se va desarrollando progresivamente en la vida humana, pero cuando la persona envejece este patrón cambia y limitan el autocuidado. Sin embargo, con el apoyo educativo se contribuye a que los adultos mayores incrementen o mantengan sus capacidades de autocuidado, solo hay que considerar las estrategias didácticas para poder motivarlos.

\section{Agradecimientos}

A los adultos mayores participantes, así mismo a autoridades del Instituto para la Atención de las y los Adultos Mayores del estado de Hidalgo que brindaron el apoyo para la realización del proyecto.

(Loredo Figueroa, Gallegos Torres, \& Xeque Morales, 2016)

\section{Referencias}

Castiblanco Amaya, M. A., \& Fajardo, R. E. (2017). Capacidad y percepción de autocuidado del adulto mayor del programa Centro día/vida, Ibagué, Colombia. Salud Uninorte, 58-65.

Garcilazo Salazar, M. E. (12 de 11 de 2019). Cybertesis. Repositorio de Tesis Digitales. Obtenido de http://cybertesis.unmsm.edu.pe/bitstream/handle/cybertesis/4131/Garci lazo_sm.

Loredo Figueroa, M., Gallegos Torres, R., \& Xeque Morales, A. (2016). Nivel de dependencia, a utocuidado y calidad de vida del asdulto mayor. Enfermería Universitaria, 159-165.
Organización Mundial de la Salud. (11 de 03 de 2015). Envejecimiento y Ciclo de Vida. Obtenido de Informe Mundial sobre el Envejecimiento y la Salud: https://apps.who.int/iris/bitstream/handle/10665/186466/97892406948 73_spa.pdf?sequence $=1$

Organización Mundial de la Salud. (05 de 02 de 2018). Envejecimiento y Salud. Obtenido de https://www.who.int/es/news-room/factsheets/detail/envejecimiento-y-salud

Raile , A. M. (2018). Modelos y teorías de enfermería. Barcelona, España: Elsevier. 